PROCEEDINGS OF THE

AMERICAN MATHEMATICAL SOCIETY

Volume 92, Number 2, October 1984

\title{
A WALLMAN-TYPE COMPACTIFICATION FOR CONVERGENCE SPACES
}

\author{
R. J. GAZIK, B. H. PARK AND G. D. RICHARDSON
}

\begin{abstract}
A compactification for any given convergence space is constructed. The compactification has the unique continuous extension property subject to reasonable assumptions on the range space. Properties of the compactification are studied.
\end{abstract}

1. Preliminaries. Let $X$ be any given convergence space and denote by $\dot{x}$ the ultrafilter on $X$ containing the set $\{x\}$. A convergence space $X$ is said to be $T_{0}$ if either $\dot{x}$ fails to converge to $y$ or $\dot{y}$ fails to converge to $x$ for each distinct $x$ and $y$ in $X$. The space is called $T_{1}$ whenever $\dot{x}$ fails to converge to $y$ for each different $x$ and $y$ in $X$ and $T_{2}$ whenever each filter on $X$ converges to at most one point in $X$. A $T_{2}$ convergence space $X$ with the property that $\operatorname{cl}_{X} \mp \rightarrow x\left(\operatorname{cl}_{\lambda X} \mp \rightarrow x\right)$ in

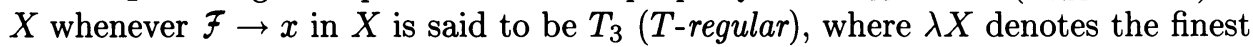
topological space coarser than $X$.

Consider a convergence space $X$. A filter on $X$ is said to be closed if it has a base of closed subsets. A closed filter $\mathcal{F}$ on $X$ is called prime closed if either $A \in \mathcal{F}$ or $B \in \mathcal{F}$ whenever $A$ and $B$ are closed subsets of $X$ and $A \cup B \in \mathcal{F}$. Let $X^{\prime}$ denote the set of all nonconvergent prime closed filters on $X$ and let $A^{*}=A \cup\left\{\mathcal{F} \in X^{\prime} \mid A \in \mathcal{F}\right\}$, where $A$ is any subset of $X$. Note that $(A \cap B)^{*}=A^{*} \cap B^{*}$ and $A^{*} \cup B^{*} \subseteq(A \cup B)^{*}$ for any subsets $A$ and $B$ of $X ; A^{*} \cup B^{*}=(A \cup B)^{*}$ whenever $A$ and $B$ are closed subsets of $X$. Denote by $\mathcal{F}^{*}$ the filter on $X^{*}$ generated by $\left\{F^{*} \mid F \in \mathcal{F}\right\}$, where $\mathcal{F}$ is any filter on $X$.

Let $X$ be any given convergence space. Define the following convergence structure on $X^{*}: \not H \rightarrow x$ iff $\mathcal{H} \geq \mathcal{F}^{*}$ (i.e. $\mathcal{F}^{*} \subseteq \not{H}$ ) for some $\mathcal{F} \rightarrow x$ in $X$; $\mathscr{H} \rightarrow \mathcal{F}, \mathcal{F} \in X^{\prime}$, iff $\mathcal{H} \geq \mathcal{F}^{*}$. Let $\phi: X \rightarrow X^{*}$ denote the map $\phi(x)=x$ for each $x \in X$. A pair $(Y, \alpha)$ is called a compactification of $X$ provided $Y$ is a compact convergence space and $\alpha: X \rightarrow Y$ is a dense embedding. A $T_{3}$ convergence space is said to be completely regular whenever it has the same ultrafilter convergence as a Tychonoff topological space. It is shown in $[\mathbf{1}]$ that a convergence space has a $T_{3}$ compactification iff it is a completely regular convergence space. The reader interested in further details is referred to [2] for a summary of results on compactifications of convergence spaces.

2. Compactification. Let us first give a characterization of prime closed filters in terms of ultrafilters. The term "ultrafilter" is abbreviated by "u.f.".

LEMMA 1. Let $₹$ denote a closed filter on the convergence space $X$. Then $₹$ is prime closed iff $\mathcal{F}=\operatorname{cl}_{\lambda X} \mathcal{G}$ for some u.f. $\mathcal{G}$ on $X$.

Received by the editors March 7, 1983 and, in revised form, October 12, 1983

1980 Mathematics Subject Classification. Primary 54A20, 54C20, 54C25, 54D35.

Key words and phrases. T-regular convergence spaces, compactifications, prime closed filters, ultraclosed filters. 
Proof. Assume that $\mathcal{F}$ is a prime closed filter on $X$ and let $C=\{\not \mid \mathcal{F}=$ $\operatorname{cl}_{\lambda X} \mathcal{H}, \mathcal{H}$ is a filter on $\left.X\right\}$. Then $C$ is a partially ordered set such that each chain in $C$ has an upper bound in $C$. Hence by Zorn's Lemma there exists a maximal element $\mathcal{G}$ in $\mathcal{C}$. Let us show that $\mathcal{G}$ is a u.f. on $X$.

Suppose that both $A$ and $A^{c}$ do not belong to $\mathcal{G}$. Then there exist two filters on $X$ containing $\{A \cap G \mid G \in \mathcal{G}\}$ and $\left\{A^{c} \cap G \mid G \in \mathcal{G}\right\}$, respectively, and each is strictly finer than $\mathcal{G}$. By the maximality of $\mathcal{G}, \operatorname{cl}_{\lambda X}(A \cap U) \notin \mathcal{F}$ and $\operatorname{cl}_{\lambda X}\left(A^{c} \cap V\right) \notin \mathcal{F}$ for some $U, V \in \mathcal{G}$. However,

$$
\operatorname{cl}_{\lambda X}(U \cap V)=\operatorname{cl}_{\lambda X}(A \cap U \cap V) \cup \operatorname{cl}_{\lambda X}\left(A^{c} \cap U \cap V\right) \in \mathcal{F}
$$

and thus either $\operatorname{cl}_{\lambda X}(A \cap U \cap V) \in \mathcal{F}$ or $\operatorname{cl}_{\lambda X}\left(A^{c} \cap U \cap V\right) \in \mathcal{F}$ since $\mathcal{F}$ is prime closed. This is contrary to the fact that $\operatorname{cl}_{\lambda X}(A \cap U)$ and $\operatorname{cl}_{\lambda X}\left(A^{c} \cap V\right)$ fail to belong to $\mathcal{F}$. Hence $\mathcal{G}$ is a u.f. on $X$.

Conversely, suppose that $\mathcal{F}=\operatorname{cl}_{\lambda X} \mathcal{G}, \mathcal{G}$ a u.f. on $X$. If $A \cup B \in \mathcal{F}, A$ and $B$ closed subsets of $X$, then $A \cup B \in \mathcal{G}$. Hence either $A \in \mathcal{G}$ or $B \in \mathcal{G}$ and thus either $A \in \mathcal{F}$ or $B \in \mathcal{F}$. Therefore $\mathcal{F}$ is a prime closed filter on $X$.

Given any convergence space $X$, the pair $\left(X^{*}, \phi\right)$ is shown to be a compactification of $X$ with a universal property. Since more than one convergence space is used, $\left(X^{*}, \phi\right)$ is denoted by $\left(X^{*}, \phi_{X}\right)$.

Proposition 1. Let $X$ be any convergence space. Then $\left(X^{*}, \phi_{X}\right)$ is a convergence space compactification of $X$. If $f: X \rightarrow Y$ is a continuous map from $X$ into a $T$-regular convergence space $Y$, then $f$ has a continuous extension $g$ such that the diagram below is commutative. Moreover, the continuous extension $g$ is unique whenever $Y^{*}$ is $T_{2}$ :

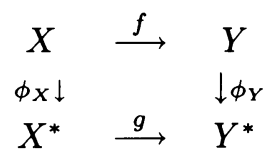

Proof. Clearly $\phi_{X}$ is a dense embedding. Let $\not$ be a u.f. on $X^{*}$; define $₹$ to be the filter on $X$ generated by $\left\{A \subseteq X \mid A\right.$ is closed and $\left.A^{*} \in \mathcal{H}\right\}$. It follows, since $(A \cup B)^{*}=A^{*} \cup B^{*}$ for closed subsets $A$ and $B$ of $X$, that $\mathcal{F}$ is prime closed. If $\mathcal{F} \rightarrow x$ in $X\left(\mathcal{F} \in X^{\prime}\right)$, then $\not H \rightarrow x(\not H \rightarrow \mathcal{F})$ in $X^{*}$. Hence $\left(X^{*}, \phi_{X}\right)$ is a compactification of $X$.

Define $g: X^{*} \rightarrow Y^{*}$ as follows: $g(x)=f(x)$ for each $x \in X$ and, for $\mp \in X^{\prime}$,

$$
g(\mathcal{F})= \begin{cases}y & \text { if } f \mathcal{F} \rightarrow y \text { in } Y, \\ \not & \text { if } f \mathcal{F} \text { fails to converge in } Y,\end{cases}
$$

where $H$ is the prime closed filter on $Y$ generated by $\{A \subseteq Y \mid A$ is closed and $\left.f^{-1}(A) \in \mathcal{F}\right\}$. Let us show that $g\left(F^{*}\right) \subseteq A^{*}$ whenever $A$ is a closed subset of $Y$ and $F=f^{-1}(A)$. Assume that $\mathcal{G} \in F^{*}$. If $f \mathcal{G} \rightarrow y$ in $Y$, then $y \in \operatorname{cl}_{Y} f(F) \subseteq A$. If $\mathcal{G}$ fails to converge in $Y$, then $g(\mathcal{G})=\mathcal{K}$, where $\mathcal{K}$ is the filter on $Y$ generated by $\left\{B \subseteq Y \mid B\right.$ is closed and $\left.f^{-1}(B) \in \mathcal{G}\right\}$. Since $\mathcal{G} \in F^{*}$, and thus $F \in \mathcal{G}$, then it follows that $A \in \mathcal{K}$. Hence $g(\mathcal{G})=\mathcal{K} \in A^{*}$ and thus $g\left(F^{*}\right) \subseteq A^{*}$. If $\mathcal{F}$ is a filter on $X$ such that $f \mathcal{F} \rightarrow y$ in $Y$, then the preceding remarks show that

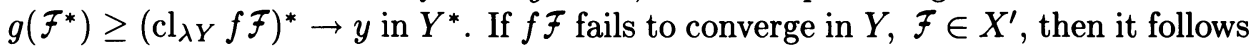
from the preceding remarks that $g\left(\mathcal{F}^{*}\right)>\mathcal{H}^{*} \rightarrow \nVdash$ in $Y^{*}$, where $\nVdash$ is defined above. Hence $g$ is a continuous map such that the diagram is commutative. 
Let us show that $g$ is the unique continuous extension making the diagram commutative whenever $Y^{*}$ is $T_{2}$. The only part needing justification is the case when-

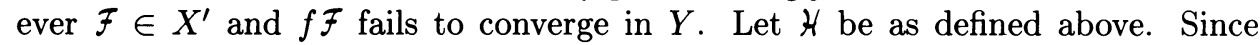
$\phi_{X}(\mathcal{F}) \rightarrow \mathcal{F}$ in $X^{*}$, then a continuous extension $g$ of $f$ making the diagram commutative must necessarily satisfy $g\left(\phi_{X} \mathcal{F}\right)=\phi_{Y} f \mathcal{F} \rightarrow g(\mathcal{F})$ in $Y^{*}$. However $f \mathcal{F} \geq \mathcal{H}$ and thus $g\left(\phi_{X} \mathcal{F}\right)=\phi_{Y} f \mathfrak{F} \geq \phi_{Y} \not H \geq H^{*} \rightarrow \not{H}$ in $Y^{*}$. Since $Y^{*}$ is $T_{2}$, then $g(\mathcal{F})=\not H$ and thus the desired extension is unique.

3. Properties of $X^{*}$. Conditions are given on $X$ which ensure certain separation axioms on $X^{*}$. The section is concluded with a remark relating $X^{*}$ to the Wallman compactification of $\lambda X$ whenever the latter is a $T_{1}$ space. The proof of the following straightforward result is omitted.

Proposition 2. Let $X$ be a convergence space. Then $X^{*}$ is $T_{0}$ iff $X$ is $T_{0}$.

A maximal closed filter, in the set-theoretic sense, on a convergence space is said to be ultraclosed. It follows from Lemma 1 that an ultraclosed filter is prime closed. However, the converse is false and this leads us to make the following definition. A convergence space is called a $U$-space if each nonconvergent prime closed filter is ultraclosed. Note that if $X$ is an infinite set with the cofinite topology, then $X$ is not a $U$-space.

Proposition 3. Suppose that $X$ is a convergence space. Then $X^{*}$ is $T_{1}\left(T_{2}, T_{3}\right)$ iff $X$ is a $T_{1}\left(T_{2}\right.$, completely regular $) U$-space.

Proof. Assume that $X^{*}$ is a $T_{i}$ space, $i=1,2,3$. Since this property is hereditary, then $X$ is also a $T_{i}$ space, $i=1,2,3$. Moreover, if $X^{*}$ is $T_{3}$, then $\left(X^{*}, \phi\right)$ is a $T_{3}$ compactification of $X$ and thus by $[\mathbf{1}$, Theorem 1] $X$ must be a completely regular convergence space. Suppose that $X^{*}$ is a $T_{1}$ space and $₹ \in X^{\prime}$. Let $\mathcal{G}$ be an ultraclosed filter on $X$ which contains $\mathcal{F}$. Then by Lemma $1, \mathcal{G} \in X^{\prime}$ and moreover $\dot{\mathcal{G}} \rightarrow \mathcal{F}$ in $X^{*}$. Since $X^{*}$ is $T_{1}$, then $\mathcal{G}=\mathcal{F}$ and hence $X$ is a $U$-space.

Conversely, suppose that $X$ is a $T_{1} U$-space. If $\phi(\dot{x}) \rightarrow \phi(y)$ in $X^{*}$, then $\phi(\dot{x}) \geq$ $\mathcal{F}^{*}$ for some $\mathcal{F} \rightarrow y$ in $X$. It follows that $\dot{x} \geq \mathcal{F}$ and thus $\dot{x} \rightarrow y$; hence $x=y$. Next, assume that $\phi(\dot{x}) \rightarrow \mathcal{F}$ in $X^{*}, \mathcal{F} \in X^{\prime}$. Then $\phi(\dot{x}) \geq \mathcal{F}^{*}$ and thus $\dot{x} \geq \mathcal{F}$. Since $X$ is a $T_{1} U$-space, then $\dot{x}=\mathcal{F}$, contrary to $\mathcal{F} \in X^{\prime}$. Suppose that $\dot{\xi} \rightarrow \phi(x)$ in $X^{*}$; then $\dot{\mathcal{F}} \geq \mathcal{G}^{*}$ for some $\mathcal{G} \rightarrow x$ in $X$. Hence $\mathcal{F} \geq \mathcal{G}$ and thus $\mathcal{F} \rightarrow x$ in $X$, contrary to $\mathcal{F} \in \bar{X}^{\prime}$. Finally, if $\dot{\mathcal{F}} \rightarrow \mathcal{G}$ in $X^{*}, \mathcal{F}$ and $\mathcal{G}$ in $X^{\prime}$, then $\dot{\mathcal{F}} \geq \mathcal{G}^{*}$ and hence $\mathcal{F} \geq \mathcal{G}$. Since $X$ is a $U$-space, then $\mathcal{F}=\mathcal{G}$ and it follows that $X^{*}$ is $T_{1}$.

A similar argument shows that $X^{*}$ is $T_{2}$ whenever $X$ is a $T_{2} U$-space. Finally, assume that $X$ is a completely regular $U$-space. Since a completely regular convergence space is $T$-regular, then if either $₹ \rightarrow x$ in $X$ or $₹ \in X^{\prime}$ we may, without loss of generality, assume that $\mathcal{F}$ is a closed filter on $X$. Furthermore, note that if $A$ is a closed subset of $X$, then $A^{*}$ is also a closed subset of $X^{*}$. Hence $\mathcal{F}^{*}$ is a closed filter on $X^{*}$ and thus $X^{*}$ is $T_{3}$.

Suppose that $X$ is a $T_{1} U$-space. Then $\lambda X$ is a $T_{1}$ topological space and hence by Proposition $3, X^{*}$ and the Wallman compactification of $\lambda X$, denoted by $W(\lambda X)$, have the same underlying set. It is straightforward to verify that if $\mathcal{F}^{*} \rightarrow s$ in $X^{*}$, then $\mathcal{F}^{*} \rightarrow s$ in $W(\lambda X)$. It follows that $\lambda X^{*}$ is finer than $W(\lambda X)$ in this case.

The authors wish to express their appreciation to the referee for making significant suggestions which resulted in major changes of the original manuscript. 


\section{REFERENCES}

1. D. C. Kent and G. D. Richardson, Regular compactifications of convergence spaces, Proc. Amer. Math. Soc. 31 (1972), 571-573.

2. __ Compactifications of convergence spaces, Internat. J. Math. Math. Sci. 2 (1979), 345-368.

Department of Mathematics and Physics, Arkansas State University, State UNIVERSITY, ARKANSAS 72467

Department of MATHEMATiCs, Gyeongsang National University, Jinju, GyEONGSANGNAMDO 620, SOUTH KOREA

Department of MATHEMATICS, EASt CAROLINA UNIVERSity, GREenVille, North CAROLINA 27834 